\title{
Cancer, Malnutrition and Cachexia: We Must Break the Triad
}

\author{
Virender Suhag ${ }^{*}$, B. S. Sunita ${ }^{2}$, Arti Sarin1, A. K. Singh ${ }^{1}$ \\ ${ }^{1}$ Army Hospital (Research \& Referral), Delhi, India \\ ${ }^{2}$ Base Hospital, Delhi, India \\ Email: *virendersuhag@gmail.com, drsunitabs@gmail.com, artisarin@gmail.com, Itcolaksingh@gmail.com
}

Received 15 January 2015; accepted 30 January 2015; published 4 February 2015

Copyright (C) 2015 by authors and Scientific Research Publishing Inc.

This work is licensed under the Creative Commons Attribution International License (CC BY). http://creativecommons.org/licenses/by/4.0/

(c) (i) Open Access

\begin{abstract}
Many factors can modify nutritional status in cancer patients, including cachexia, nausea and vomiting, decreased caloric intake or oncologic treatments causing malabsorption. The cachexiaanorexia syndrome is a complex metabolic syndrome associated with cancer and some other palliative conditions characterized by involuntary weight loss involving fat and muscle, anorexia, early satiety, fatigue and weakness due to shifts in metabolism caused by tumour by-products and cytokines. Cachexia is a distressing and debilitating condition, affecting significant numbers of patients with advanced disease and is the primary cause of death in about $20 \%$ of all patients with cancer. Though cachexia is most commonly associated with particular tumours, such as head and neck, gastrointestinal tract, pancreas, central nervous system and lung, it may affect any patient with any tumour at any site; no patient and no tumour are excluded. Current treatment for principally depends on its prevention rather than reversing the present disease state, and the clinical results are far from being satisfactory. A careful decision based on good clinical judgement is necessary before deciding to start either enteral or parenteral nutrition, to avoid a useless, costly and difficult treatment. Treatment should be directed toward improvement in the quality of life of the patient and should often include nutritional counseling. It should take into consideration both disease and treatment related factors as well as the cachexia syndrome itself.
\end{abstract}

\section{Keywords}

Cancer, Malnutrition, Cachexia, Nutritional Support

\section{Introduction}

Cancer as a disease and malnutrition are closely linked and can rapidly develop in to a vicious circle, in which ${ }^{*}$ Corresponding author.

How to cite this paper: Suhag, V., Sunita, B.S., Sarin, A. and Singh, A.K. (2015) Cancer, Malnutrition and Cachexia: We Must Break the Triad. International Journal of Medical Physics, Clinical Engineering and Radiation Oncology, 4, 64-70.

http://dx.doi.org/10.4236/ijmpcero.2015.41009 
disease causes decreased desire for eating, malabsorption and increased loss of nutrients, which in turn cause increased susceptibility to complications. These complications further impair the well-being of patient, including fatigue, decreased mobility, poor response to treatment and further complications, which results in further decreased nutritional status. Therefore, cancer malnutrition and cachexia is a self-perpetuating condition; and the breakage of this circle can improve treatment outcomes and quality of life measures, which can be achieved through providing the appropriate medications and nutritional support [1] [2]. The term cachexia derives from the Greek words "kakos" and "hexis" for "bad condition". It is a progressive wasting condition that is encountered not only in cancer but also in other life-threatening diseases such as AIDS, rheumatoid arthritis, chronic obstructive pulmonary diseases, and organ failure. The significant weight loss resulting from this condition causes impairment of immune function, poor outcome of chemotherapy, fatigue, and markedly reduced quality of life [2]. Cachexia may be defined as a multifactorial syndrome characterized by an ongoing loss of skeletal muscle mass, with or without loss of fat mass, which cannot be fully reversed by conventional nutritional support and leads to progressive functional impairment. Cachexia pathophysiology is characterized by a negative protein and energy balance driven by a variable combination of reduced food intake and abnormal metabolism [3]-[5].

For many years, the terms "malnutrition", "weight loss" and "cachexia" have been used interchangeably in the scientific literature [6] [7]. However, when referring to cancer patients, we prefer to use the term "cachexia", mainly for two reasons. Firstly, the term "malnutrition" in a broad sense would also include conditions of overweight and obesity and, second and more importantly, it suggests that the cause of weight loss is mainly a reduced intake of food, whereas in cancer patients quite often there is a combination of poor intake and metabolic alterations. Furthermore, the term "malnutrition" would imply that an adequate nutritional support is able to fully reverse the state of weight loss or emaciation, whereas in the effect of a standard optimized nutritional support can only prevent a further deterioration of the general status of the patients and, for a successful outcome, nutritional support needs to be combined, at an early stage, with anabolic/anticatabolic agents [4] [8].

Cancer cachexia and malnutrition affect $40 \%$ to $85 \%$ of all terminally ill patients, accounting more than $20 \%$ of all cancer deaths. Current treatment for principally depends on its prevention rather than reversing the disease state. Currently it appears to be logical to accept as an early disease step which is triggered at the beginning of disease when even no weight loss is detected, and fight against it before its advance to an irreversible condition [2] [9]. Dietary counseling, recommended for patients at risk of malnutrition, should be introduced early in close collaboration with the patient. Administering oral nutritional supplements in selected malnourished patients has been shown to lead to a reduction in mortality by decreasing complications and the length of hospital stay. Supplementation with enteral nutrition has demonstrated an increase in appetite, energy intake, nutritional status, and, above all, reduced gastrointestinal toxicity from cancer treatments due to a better response to them. Supplementation with home parenteral nutrition in aphagic and terminal patients has shown improved quality of life, energy balance, body composition and prolonged survival [10]. This review article will briefly summarize the etiopathology of malnutrition, its effect on patient's quality of life, nutritional screening, nutritional assessment, management of cachexia, pharmacological intervention, rehabilitation and role of nutritional interventions in palliative management.

\section{Etiopathology of Malnutrition \& Weight Loss}

Weight loss in cancer patients is due to diverse factors, among them the production of inflammatory and catabolic mediators playing important roles. Such markers include acute-phase proteins, interleukin-6 (IL-6), or the ubiquitine-proteasome complex whose activity may be increased by the neoplasm itself, particularly in some rapidly growing tumors such as those of the pancreas or the lungs. Inflammation plays a pivotal role in the pathogenesis of cachexia: an imbalance between pro-inflammatory (e.g., tumor necrosis factor- $\alpha$ [TNF- $\alpha$ ], IL-1, IL-6, interferon- $\gamma$ [IFN- $\gamma]$ ) and anti-inflammatory (e.g., IL-4, IL-12, IL-15) cytokines is currently considered to contribute to cachexia development and progression. Pre-cachexia immediately precedes cachexia and can be diagnosed in the presence of all the following criteria: 1) underlying chronic disease; 2) unintentional weight loss $>=5 \%$ of usual body weight during the last 6 months; 3 ) chronic or recurrent systemic inflammatory response; and 4) anorexia or anorexia-related symptoms.

The neoplastic mass can represent a possible mechanical obstruction to the way in the digestive tract, causing dysphagia and impaired swallowing (head-neck, esophageal cancer, or mediastinal masses), early satiety, nausea, vomiting (gastric and small bowel tumors), abdominal pain for intestinal sub-occlusion or occlusion (small and 
large bowel, peritoneal carcinomatosis). Moreover, the tumor can interfere with organ function, for example, causing diarrhea (pancreatic and biliary cancer) as a result of the lack of digestive enzymes. The presence of continuous or occasional pain during eating and digestion may represent another important factor limiting, quantitatively and qualitatively, oral intake [2] [11].

Furthermore, following surgery for cancer removal, gastrointestinal changes can affect the digestive processes causing, for example, early satiety, dumping syndrome, or diarrhea. Each condition requires specific dietetic interventions aimed at progressive nutritional rehabilitation. Anticancer treatments can also be a major cause of malnutrition. Chemotherapy causes nausea, vomiting, abdominal cramping and bloating, mucositis, paralytic ileus and even malabsorption. Despite the recent advent of antiemetic drugs and the optimization of their administration schedule, vomiting remains an important cause of malnutrition in cancer. Various antineoplastic agents, such as fluorouracil, adriamycin, methotrexate and cisplatin, induce severe gastrointestinal complications [2]. Malnutrition and cachexia can negatively affect the clinical decision to resect the tumor, and can increase the incidence of postoperative complications, such as delayed wound healing, dehiscence of anastomosis, morbidity, and mortality. In addition, malnutrition is an independent risk factor for quality of life. Malnutrition impairs the immune status and reduces the body's defense against infectious diseases. In light of these possible complications, malnutrition represents a poor prognostic factor and, as such, should be prevented or detected as early as possible [2] [12].

\section{Interactions between Quality of Life (QoL) and Nutritional Status}

Health-related QoL is a multidimensional concept which quantifies the psychological, physical and social effects of an illness and its therapy. In cancer patients, the health status is well reflected in the measured QoL, which is largely influenced by nutritional aspects. Cancer and its treatment result in severe biochemical and physiological alterations associated with a deterioration of QoL. These metabolic changes lead to decreased food intake and promote wasting [13]. Depending on the type of cancer treatment (either curative or palliative), the clinical condition of the patient and nutritional status, adequate and patient-tailored nutritional intervention should be prescribed (diet counseling, oral supplementation, enteral or total parenteral nutrition). Such an approach, which should be started as early as possible, reduces or even reverses their poor nutritional status, improves their performance status and consequently their QoL. Assessment of QoL should be considered in the evaluation of any nutritional support to optimize its benefits in regards to the needs and expectations of the patient. The inadequate nutritional status and cancer anorexia-cachexia syndrome related to it are clinically relevant since the response to antineoplastic measures, such as radiation and chemotherapy, may be diminished, their side effects aggravated and patient QoL and prognosis negatively affected [2] [3].

\section{Nutritional Screening \& Nutritional Assessment}

Nutritional screening aims to identify patients at risk of malnutrition in a simple and non-invasive way. It is followed by full evaluation (nutritional assessment) to confirm and classify the degree of malnutrition. These two steps allow early identifying and treating patients with malnutrition or periodically follow-up those at high nutritional risk, to face any nutritional challenges before significant weight loss or before other clinical/biological signs of malnutrition appear. Due to time restrictions of healthcare professionals and cost implications, nutritional screening needs to be feasible, speedy, inexpensive, and non-invasive [2] [14]. In all cancer patients primary assessment of nutritional status and energy intake should be done ideally at the beginning of the disease. It is important to measure weight at regular intervals. Undernutrition is likely when a patient has lost more than $10 \%$ of his/her actual weight over the last 6 months or more than $5 \%$ over the last 3 months or when BMI is lower than $18.5 \mathrm{~kg} / \mathrm{m}^{2}$ [15] [16]. Careful clinical history record and meticulous symptom assessment are the first steps to become sympathetic with the patient and to understand his/her conditions in more detail. Another major step is the compilation, with the help of a dietitian, of a detailed food record in the days between chemotherapy cycles (when patients are feeling better) and the days of treatment; the compilation of questionnaires about patients' daily activities (ability to work, social life, autonomy in personal hygiene, time spent in bed) should also be recommended [2] [16].

Several clinical and laboratory scores have been developed to identify malnourished patients and/or patients at risk of undernutrition. One of the best evaluated scores is the "Subjective Global Assessment" (SGA), which is based exclusively on anamnestic criteria like weight change, GI symptoms, performance status, nutrient 
requirement and a simple physical examination (oedemas, ascites, muscle mass, fat mass). Thereby, nutritional status is subjectively classified as A = well-nourished, B = moderately malnourished/at risk of undernutrition, $\mathrm{C}=$ severely malnourished. ESPEN, the European Society of Clinical Nutrition and Metabolism, has recommended two simple but validated screening questionnaires, the Nutritional Risk Screening 2002 (NRS2002) and the Malnutrition Universal Screening Tool (MUST) [15] [17] [18].

\section{Treatment of Malnutrition}

Both parenteral and enteral nutrition are now increasingly integrated within the main oncologic strategy with the aim of making surgery, chemotherapy and radiation therapy more safe and effective. This requires a better awareness of the inherent risk of starvation and undernutrition by the surgeons, medical oncologists and radiation oncologists, the ability to implement a policy of nutritional screening of cancer patients and to propose them the nutritional support in a single bundle together with the oncologic drugs. Four different areas of nutritional intervention are now recognized which parallel the evolutionary trajectory of patients with tumour: the perioperative nutrition in surgical patients, the permissive nutrition in patients receiving chemotherapy and/or radiation therapy and the home parenteral nutrition which may be total (in aphagic-obstructed-incurable patients) or supplemental (in advanced weight-losing anorectic patients) [19]. The therapeutic goal for cancer patients is the improvement of function and outcome by 1) preventing and treating undernutrition; 2) enhancing antitumor treatment effects; 3) reducing adverse effects of antitumor therapies; and 4) improving QoL [15]. Nutritional therapy should be initiated when undernutrition already exists or when it is anticipated that the patient may be unable to eat for more than 7 days. Enteral nutrition should also be initiated when an inadequate food intake ( $<60 \%$ of estimated energy expenditure) is anticipated for more than 10 days. It should substitute the difference between actual intake and calculated requirements [15] [16]. Appropriate treatment of cancer cachexia should address the following conditions: inflammatory state, nutritional disorder, metabolic derangements, immunological defects, poor quality of life, and, in particular, fatigue. Accordingly, treatment for cancer cachexia should include as primary endpoints the following variables: an increase in lean body mass and functional activity; a decrease in resting energy expenditure; and improvement of fatigue [20] [21].

In patients with head/neck cancer on radiotherapy or combined chemo- and radiotherapy, weight loss generally commences in the second week of therapy. Considering that the gastrointestinal tract distal of the tumor and the treatment field is usually functional, enteral rather than parenteral feeding yields safer and more physiological results. The positioning of a nasogastric tube (NGT) for enteral nutrition can be advisable when a temporary dysphagia is anticipated (during and soon after radiation therapy). On the other hand, at stage 3 or 4 of the disease, the prophylactic placement of a percutaneous gastrostomy (PEG) for early enteral nutrition can be a reasonable approach. While NGT positioning has generally been recommended when nutritional support is required for a short period of time (less than 1 month), PEG tubes are the better choice for prolonged nutritional support [2] [3].

In malnourished patients who undergo radiotherapy as single or concomitant therapy for gastrointestinal neoplasms, abdominal pain, nausea, vomiting, or diarrhea may not permit an adequate oral support and intestinal absorption. Therefore, a parenteral nutritional therapy through peripheral veins or preferably through a pre-existing central venous catheter could be necessary [2]. The treatment aims for total parenteral nutrition (TPN) in cancer patients are as follows. 1) TPN should stabilize the nutritional state and prevent or reduce progressive weight loss; 2) TPN should maintain or improve the QoL; 3) TPN may increase the effectivity and reduce the side effects of anticancer therapies. The majority of cancer patients requiring long-term TPN are cachectic and hypophagic because of (subacute) intestinal obstruction due to peritoneal carcinomatosis. This condition is often associated with expansion of extracellular water volume, and an overzealous administration of glucose may easily precipitate a peritoneal effusion which consequently forces withdrawal of the intravenous nutrition [3].

Enteral and parenteral nutrition confer a number of risks, including the physiologic stress and discomfort associated with the placement of a feeding tube or central line and complications involved in the placement or in nutrition. Infection is the most common complication of both types of nutritional support and occurs frequently with parenteral nutrition because of the high nutrient value of the infusion. Although usually easily treated, these infections often require hospitalization and insertion of a new catheter, and may lead to complications, such as subacute bacterial endocarditis. Other common complications of enteral and parenteral nutrition include metabolic problems, such as hyperglycemia and fluid, and electrolyte imbalances, diarrhea from enteral feeding and hepatic abnormalities from parenteral feeding. In the terminally ill, both types of nutrition cause fluid overload, worsening edema or shortness of breath [3]. 


\section{Pharmacological Intervention}

Progestagens were the first agents used for the treatment of cancer cachexia and are currently the favored agents approved in Europe for its treatment. Megestrol acetate and medroxyprogesterone acetate (MPA) are equivalent in terms of effectiveness in the treatment of cancer cachexia [22]. The positive effects of megestrol acetate on weight and well-being have been observed at oral doses in the range of $160-1600 \mathrm{mg} / \mathrm{day}$. MPA has been used at doses in the range of $300-4000 \mathrm{mg} /$ day. Both megestrol acetate and MPA may have adverse effects, including an increased risk of thromboembolic events, peripheral edema, breakthrough bleeding, hyperglycemia, hypertension, and Cushing's syndrome [23] [24]. Several randomized, placebo-controlled studies have shown that corticosteroids achieve a limited (up to one month) improvement in appetite, food intake, nausea, and feeling of well-being. The rapid beneficial effect of corticosteroids on mood and behavior significantly improves quality of life [25] [26]. The mechanism of action of corticosteroids in cancer cachexia is not well understood, although inhibition of prostaglandin activity and suppression of IL-1 and TNF- $\alpha$ production and release are the most well recognized targets. The development of selective COX-2 inhibitors has resulted in safer modulation of cancer-associated inflammation, and these agents could help alleviate or control cancer cachexia. Moreover, the selective COX-2 inhibitors have shown potent inhibitory and preventive effects on tumor growth in animal models; therefore, their antineoplastic activity may contribute to their ability to counteract cachexia [27]. The use of eicosapentaenoic acid (EPA) containing protein and energy-dense ONS (EPA-ONS) has been shown to reduce weight loss, increase lean body mass, improve functional capacity, nutritional status and QoL, however not to any greater degree than conventional supplements. Anabolic agents such as ghrelin and selective androgen receptor modulators are under late-phase clinical testing and hold promise as new therapies [28] [29]. Review of scientific data suggests that a single therapy may not be the best approach in treating cachexia and treatment regimens involving different combinations are more likely to be successful. Indeed the combination of different nutraceuticals with a high energy diet has led to beneficial effects in experimental animals. An interesting phase II study, involving the administration of anti-oxidants, pharmaconutritional support, progestagen and anti-cyclooxygenase-2 drugs, showed both efficacy and safety in the treatment of patients with advanced cancer of different sites suffering from cachexia” [30].

\section{Cancer Rehabilitation}

The clinical evaluation of patients with cancer cachexia has to include nutritional assessment. Depending on the needs of the patient and his/her family, members of the rehabilitation team may include any or all involved physicians, oncology nurses, dietitians, physiotherapists, and psychologists. The team has to address the potential rehabilitation needs of the individual from cancer diagnosis to rehabilitation, covering the following objectives: pain-killer therapy, nutritional and psychosocial support, and optimization of physical and social functioning. The implementation of a nutritional surveillance could enable rapid treatment of symptoms (anorexia, dysphagia, nausea, vomiting, constipation, tiredness, etc.), which could have a role in the malnutrition process. The first step of cancer rehabilitation is the primary support to the negative effects of cancer disease and its therapy. Oncologists have to be encouraged to share their work with other team specialists: anesthesiologists for pain therapy, nutritionists for nutritional support, and physical and rehabilitation medicine specialists. Physiotherapists, dietitians, and specialized nurses complete the team. All these figures have to cooperate to symptoms' management of cancer patients. It is necessary to achieve early treatment of malnutrition to avoid a gradual progression to cachexia, an irreversible condition. Patients have to be treated when an effective intervention is still possible, before cachexia manifests. It will be meaningless and unethical to treat the irreversible phase of cachexia, when the patient is almost at the end of life. In that condition, hydration is indicated. Malnutrition and above all cachexia have to be prevented and/or followed-up from the first diagnosis of cancer [2] [31] [32].

\section{Influence of Nutritional Intervention on Palliative Treatment}

Palliative care is focused on maintaining adequate hydration, alleviating or controlling symptoms (e.g., nausea and vomiting) and maintaining body weight and composition. The decision for or against artificial nutrition (enteral or parenteral) is especially difficult in palliative care patients who have entered their final phase of life. Quality of life is certainly the most important criteria in these patients and is mainly dependent on nutritional status. In the past nutritional intervention focused only on one specific intervention like dietary counseling, sip feeding or artificial nutrition. This approach has not been shown conclusively to be beneficial. The specialized 
approach meet the complex situation has been found to be better than a specific "single intervention". Strains through the disease itself but also through therapeutic measures have a major impact on QOL. Therefore, the actual current situation of the patient should be newly evaluated regularly. Home enteral and parenteral nutrition not only allows the patients to be at home, but also is more cost-effective than in-patient care [15] [33] [34].

If life expectancy is $<3$ months and/or Karnofsky-Index $<50 \%$, the indication for parenteral nutrition should be thoroughly reviewed. However, implementation of home parenteral nutrition should be always seen in context of the patients' wish, medical condition, family and therapeutic objectives. During the very final phase of life, hydration with $1000-1500 \mathrm{~mL}$ (i.v. or s.c.) of isotonic saline is often sufficient. At the very final time of life, at least every decision on therapeutic interventions should base on the individual situation and needs of the patients [15].

\section{Conclusion}

Cancer cachexia is a complex syndrome characterized by a chronic, progressive, involuntary weight loss which is poorly or only partially responsive to standard nutritional support and it is often associated with anorexia, early satiety and asthenia. It adversely affects patient outcome and is associated with poor response to treatment against cancer, susceptibility to treatment related toxicity and complications, poor quality of life and mortality. Current treatment for cancer cachexia principally depends on its prevention rather than reversing the disease state. An interdisciplinary approach (oncologist, nutritionist, nurse, dietitian, physical therapist, psychologist, etc.) is necessary for patients who are experiencing loss of physiological or biological function, fatigue, malnutrition, psychological distress, and other symptoms as a result of cancer disease or its treatment. It is necessary to make all healthcare professionals aware of the opportunity to screen cancer patients and identify those at risk of malnutrition. It is a duty of any physician and the patients' right to improve preventive actions and intervene early during cancer treatment and progression of the disease. Finally, prevention of the treatment related morbidities including nausea, vomiting, dysphagia, mucositis, diarrhea, pain, and depression may positively impact the patients QoL and treatment results against both cancer itself and related cachexia.

\section{References}

[1] Topkan, E., Yavuz, A.A. and Ozyilkan, O. (2007) Cancer Cachexia: Pathophysiologic Aspects and Treatment Options. Asian Pacific Journal of Cancer Prevention, 8, 445-451.

[2] Santarpia, L., Contaldo, F. and Pasanisi, F. (2011) Nutritional Screening and Early Treatment of Malnutrition in Cancer Patients. Journal of Cachexia, Sarcopenia and Muscle, 2, 27-35. http://dx.doi.org/10.1007/s13539-011-0022-x

[3] Akbulut, G. (2011) New Perspective for Nutritional Support of Cancer Patients: Enteral/Parenteral Nutrition. Experimental and Therapeutic Medicine, 2, 675-684.

[4] Bozzetti, F. (2013) Nutritional Support of the Oncology Patient. Critical Reviews in Oncology \& Hematology, 87, 172-200. http://dx.doi.org/10.1016/j.critrevonc.2013.03.006

[5] Bosaeus, I. (2008) Nutritional Support in Multimodal Therapy for Cancer Cachexia. Supportive Care in Cancer, 16, 447-451. http://dx.doi.org/10.1007/s00520-007-0388-7

[6] Bennani-Baiti, N. and Walsh, D. (2009) What Is Cancer Anorexia-Cachexia Syndrome? A Historical Perspective. The Journal of the Royal College of Physicians of Edinburgh, 39, 257-262.

[7] Yavuzsen, T., Walsh, D., Davis, M.P., Kirkova, J., Jin, T., LeGrand, S., et al. (2009) Components of the AnorexiaCachexia Syndrome: Gastrointestinal Symptom Correlates of Cancer Anorexia. Supportive Care in Cancer, 17, 15311541. http://dx.doi.org/10.1007/s00520-009-0623-5

[8] Patra, S.K. and Arora, S. (2012) Integrative Role of Neuropeptides and Cytokines in Cancer Anorexia-Cachexia Syndrome. Clinica Chimica Acta, 413, 1025-1034. http://dx.doi.org/10.1016/j.cca.2011.12.008

[9] Holmes, S. (2011) Nutrition in the Care of Patients with Cancer Cachexia. British Journal of Community Nursing, 16, 314-318. http://dx.doi.org/10.12968/bjen.2011.16.7.314

[10] Paccagnella, A., Morassutti, I. and Rosti, G. (2011) Nutritional Intervention for Improving Treatment Tolerance in Cancer Patients. Current Opinion in Oncology, 23, 322-330. http://dx.doi.org/10.1097/CCO.0b013e3283479c66

[11] Mondello, P., Mian, M., Aloisi, C., Famà, F., Mondello, S. and Pitini, V. (2014) Syndrome: Pathogenesis, Diagnosis, and New Therapeutic Options. Nutrition and Cancer, 16, 1-15.

[12] Penet, M.F., Winnard, P.T., Jacobs, M.A. and Bhujwalla, Z.M. (2011) Understanding Cancer-Induced Cachexia: Imaging the Flame and Its Fuel. Current Opinion in Supportive and Palliative Care, 5, 327-333. 
http://dx.doi.org/10.1097/SPC.0b013e32834c49ba

[13] Laviano, A., Meguid, M.M., Inui, A., Muscaritoli, M. and Fanelli, R.F. (2005) Therapy Insight: Cancer AnorexiaCachexia Syndrome-When All You Can Eat Is Yourself. Nature Clinical Practice Oncology, 2, 158-165. http://dx.doi.org/10.1038/ncponc0112

[14] Campos-Ferraz, P.L., Andrade, I., Neves, W.D., Hangai, I., Rodrigues-Alves, C.R. and Lancha, A.H. (2014) An Overview of Amines as Nutritional Supplements to Counteract. Journal of Cachexia, Sarcopenia and Muscle, 5, 105-110.

[15] Ockenga, J. and Valentini, L. (2005) Review Article: Anorexia and Cachexia in Gastrointestinal Cancer. Alimentary Pharmacology \& Therapeutics, 22, 583-594. http://dx.doi.org/10.1111/j.1365-2036.2005.02628.x

[16] Barrera, R. (2002) Nutritional Support in Cancer Patients. Journal of Parenteral and Enteral Nutrition, $26,63-71$. http://dx.doi.org/10.1177/014860710202600516

[17] Bozzetti, F., Mariani, L., Lo Vullo, S., Amerio, M.L., Biffi, R., Caccialanza, G., et al. (2012) The Nutritional Risk in Oncology: A Study of 1,453 Cancer Outpatients. Supportive Care in Cancer, 20, 1919-1928. http://dx.doi.org/10.1007/s00520-012-1387-x

[18] Bozzetti, F., Arends, J., Lundholm, K., Micklewright, A., Zurcher, G. and Muscaritoli, M. (2009) ESPEN Guidelines on Parenteral Nutrition: Non-Surgical Oncology. Clinical Nutrition, 28, 445-454. http://dx.doi.org/10.1016/j.clnu.2009.04.011

[19] Bozzetti, F. (2011) Nutritional Support in Oncologic Patients: Where We Are and Where We Are Going. Clinical Nutrition, 30, 714-747. http://dx.doi.org/10.1016/j.clnu.2011.06.011

[20] Penna, F., Minero, V.G., Costamagna, D., Bonelli, G., Baccino, F.M. and Costelli, P. (2010) Anti-Cytokine Strategies for the Treatment of Cancer-Related Anorexia and Cachexia. Expert Opinion on Biological Therapy, 10, 1241-1250. http://dx.doi.org/10.1517/14712598.2010.503773

[21] Mariani, L., Lo Vullo, S. and Bozzetti, F., on behalf of the SCRINIO Working Group (2011) Weight Loss in Cancer Patients: A Plea for a Better Awareness of the Issue. Supportive Care in Cancer, 20, 301-309. http://dx.doi.org/10.1007/s00520-010-1075-7

[22] Pascual López, A., Roqué i Figuls, M., Urrútia Cuchi, G., Berenstein, E.G., Almenar Pasies, B., Balcells Alegre, M., et al. (2004) Systematic Review of Megestrol Acetate in the Treatment of Anorexia-Cachexia Syndrome. Journal of Pain and Symptom Management, 27, 360-369. http://dx.doi.org/10.1016/j.jpainsymman.2003.09.007

[23] von Haehling, S. and Anker, S.D. (2014) Treatment of Cachexia: An Overview of Recent Developments. International Journal of Cardiology, 15, 866-872.

[24] Ruiz Garcia, V., López-Briz, E., Carbonel, S.R., Gonzalvez-Perales, J.L. and Bort-Marti, S. (2012) Megestrol Acetate for Treatment of Anorexia-Cachexia Syndrome. The Cochrane Database of Systematic Reviews, 3, CD004310.

[25] Yennurajalingam, S. and Bruera, E. (2014) Role of Corticosteroids for Fatigue in Advanced Incurable Cancer: Is It a “Wonder Drug” or "Deal with the Devil”. Current Opinion in Supportive \& Palliative Care, 8, 346-351. http://dx.doi.org/10.1097/SPC.0000000000000093

[26] Strasser, F. and Bruera, E.D. (2002) Update on Anorexia and Cachexia. Hematology/Oncology Clinics of North America, 16, 589-617. http://dx.doi.org/10.1016/S0889-8588(02)00011-4

[27] Mantovani, G., Madeddu, C. and Macciò, A. (2013) Drugs in Development for Treatment of Patients with Cancer-Related Anorexia and Cachexia Syndrome. Drug Design, Development and Therapy, 7, 645-656. http://dx.doi.org/10.2147/DDDT.S39771

[28] Balstad, T.R., Kaasa, S. and Solheim, T.S. (2014) Multimodal Nutrition/Anabolic Therapy for Wasting Conditions. Current Opinion in Clinical Nutrition and Metabolic Care, 17, 226-235. http://dx.doi.org/10.1097/MCO.0000000000000045

[29] Ströhle, A., Zänker, K. and Hahn A. (2010) Nutrition in Oncology: The Case of Micronutrients. Oncology Reports, 24, 815-828. http://dx.doi.org/10.3892/or.2010.815

[30] Argilés, J.M., Olivan, M., Busquets, S. and López-Soriano, F.J. (2010) Optimal Management of Cancer AnorexiaCachexia Syndrome. Cancer Management and Research, 2, 27-38. http://dx.doi.org/10.2147/CMAR.S7101

[31] Bruera, E. (2013) Emerging Evidence on Psychosocial Effects of Cancer Cachexia: Commentary on Oberholzer et al. Journal of Pain and Symptom Management, 46, 76. http://dx.doi.org/10.1016/j.jpainsymman.2013.05.005

[32] Bruera, E. and Hui, D. (2013) Palliative Care Research: Lessons Learned by Our Team over the Last 25 Years. Palliative Medicine, 27, 939-951. http://dx.doi.org/10.1177/0269216313477177

[33] Muliawati, Y., Haroen, H. and Rotty, L.W. (2012) Cancer Anorexia-Cachexia Syndrome. Acta Medica Indonesiana, 44, 154-162.

[34] Acreman, S. (2009) Nutrition in Palliative Care. British Journal of Community Nursing, 14, 427-428, 430-431. http://dx.doi.org/10.12968/bjen.2009.14.10.44494 
Scientific Research Publishing (SCIRP) is one of the largest Open Access journal publishers. It is currently publishing more than 200 open access, online, peer-reviewed journals covering a wide range of academic disciplines. SCIRP serves the worldwide academic communities and contributes to the progress and application of science with its publication.

Other selected journals from SCIRP are listed as below. Submit your manuscript to us via either submit@scirp.org or Online Submission Portal.
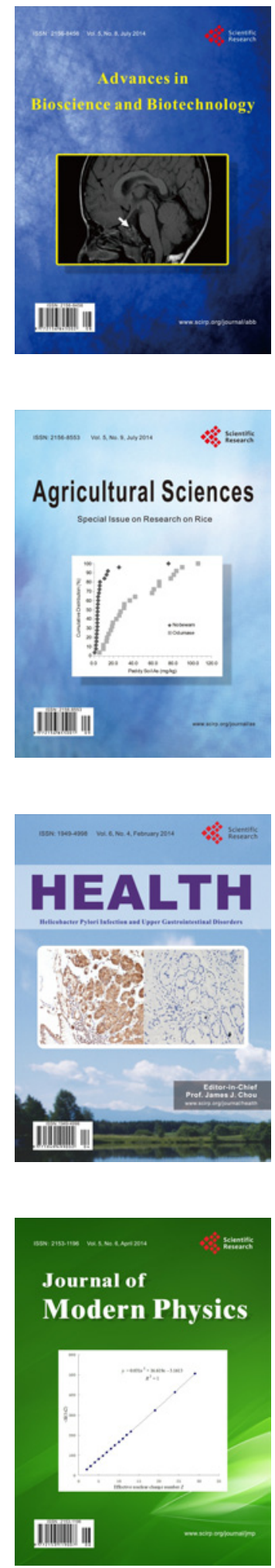
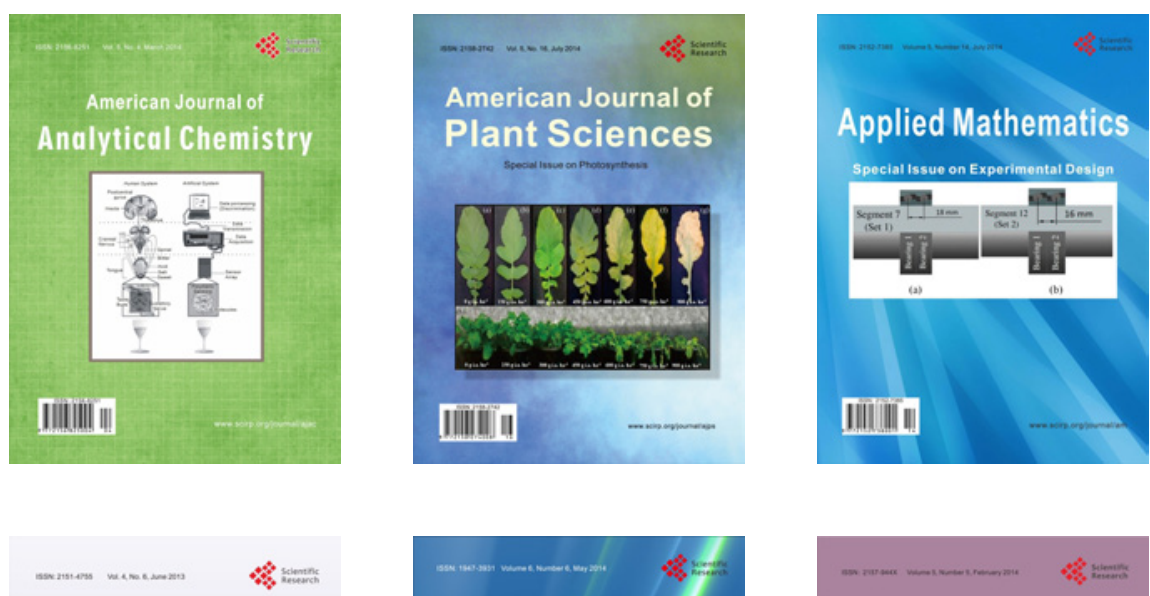

Creative Education
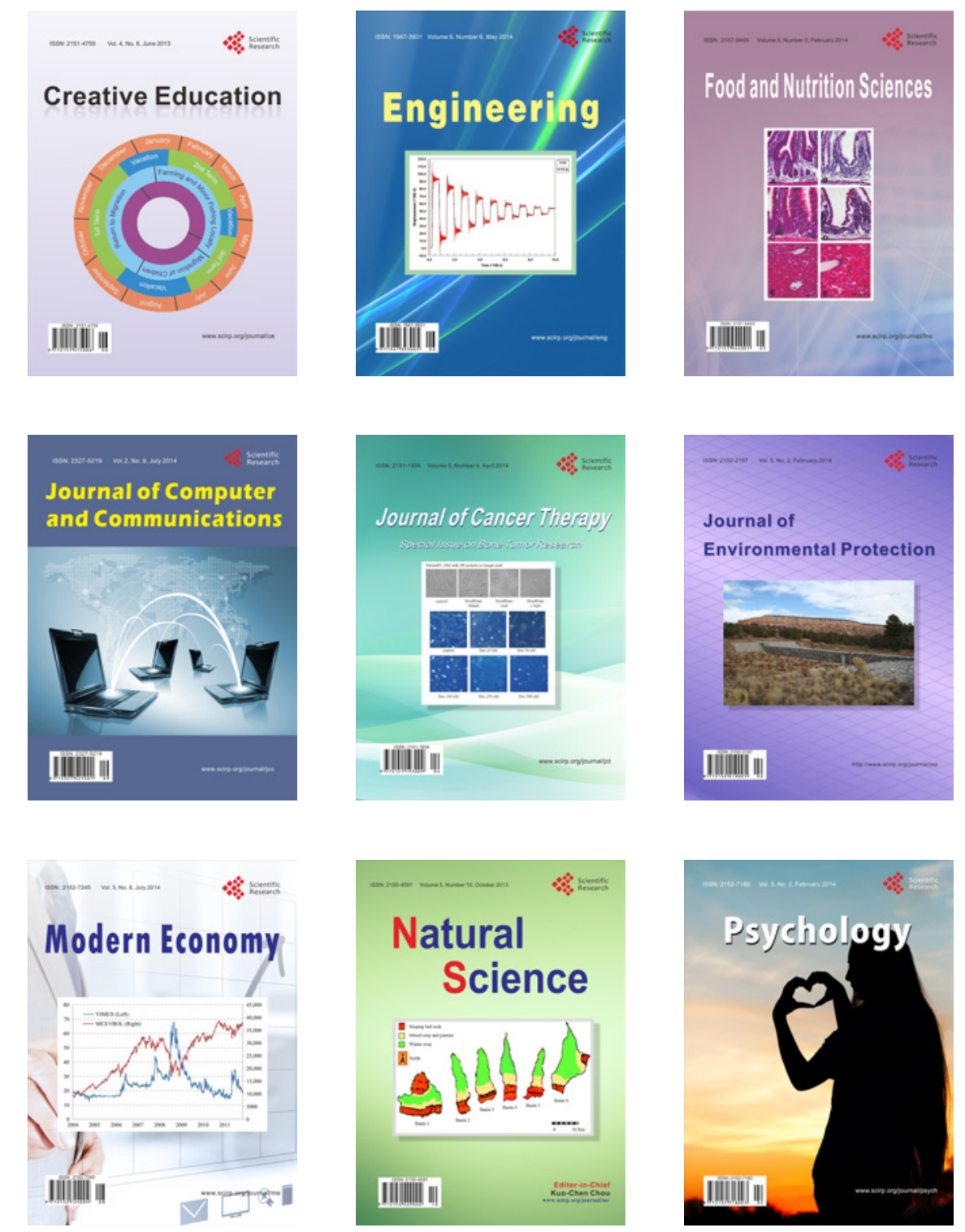